\title{
A Hydraulic Soft Microhand with an Application of Live Insect Manipulation
}

Sina Baghbani Kordmahale ( $\nabla$ sina_baq.k@tamu.edu )

Texas A\&M University

Jian Qu

Texas A\&M University

Anastasia Hanifah Muliana

Texas A\&M University

Jun Kameoka

Texas A\&M University

\section{Research Article}

Keywords: soft grippers, Polydimethylsiloxane, micro/bio manipulator

Posted Date: September 14th, 2021

DOI: https://doi.org/10.21203/rs.3.rs-899495/v1

License: @ (i) This work is licensed under a Creative Commons Attribution 4.0 International License. Read Full License 


\section{A Hydraulic Soft Microhand with an Application of Live Insect Manipulation}

Sina Baghbani Kordmahale ${ }^{1^{*}}$, Jian Qu ${ }^{3}$, Anastasia Hanifah Muliana ${ }^{3}$, Jun Kameoka ${ }^{1,2}$

${ }^{1}$ Department of Electrical Engineering and computer science, Texas A\&M University, College Station, Texas, USA. ${ }^{2}$ Department of Material Science, Texas A\&M University, College Station, Texas, USA. ${ }^{3}$ Department of Mechanical Engineering, Texas A\&M University, College Station, Texas, USA.

Correspondence and requests for materials should be addressed to S.BK (email: sina baq.k@tamu.edu)

Abstract:

We have developed micro scale hydraulic/pneumatic soft grippers and demonstrated the handling of an insect. These grippers are built on Polydimethylsiloxane (PDMS) with the soft material casting technique to form three finger-like columns, which are placed on a circular membrane. The fingers have a length of $1.5 \mathrm{~mm} / 2 \mathrm{~mm}$ and a diameter of $300 \mu \mathrm{m}$ each; the distance between two fingers is $600 \mu \mathrm{m}$ of center-tocenter distance. Membrane is built as a $150 \mu \mathrm{m}$ soft skin on the top of a cylindrical void. Applying a pressure difference between the interior of the void and the exterior can bend the membrane. Bending the membrane causes the motion of opening/closing of the gripper, and as a result, the three fingers can grip an object or release it. The PDMS was characterized and the experimental results were used later in Abaqus software to simulate the gripping motion. The produced force and range of deformation of the grippers were investigated by simulation and experiment. The results of the simulation agrees with the experiments. The maximum $543 \mu \mathrm{N}$ force was measured for the microfluidic compatible microgrippers. Using this microhand gripper, the an ant was manipulated successfully without any damage. Results showed fabricated devices have great potential as a micro/bio manipulator.

\section{Introduction:}

The evolution in robotics in the past several decades opened new opportunities in various fields like the fields of surgery, biological studies, and small objects manipulation in industry [1],[2],[3],[4],[5],[6]. Minimally Invasive surgery (MIS) is highly depending on robotic technology to minimize patient trauma and improve clinical outcomes [7]. More clinical uptake of MIS needs more flexible robots to provide higher dexterity to the surgeon, minimize the blueprint, and delicate robot-tissue contact [7],[8]. Also, Biological specimen collection and handling of delicate organisms in various environments, and with different sizes need to be performed successfully by using robotic manipulators [9]. Most of commercially available manipulation systems with their rigid structures are not suitable to handle delicate tissues and specimen [9], [10]. In the industry, precisely controlled grippers are essential and are widely operated to execute multiple tasks under various environment [11]. The evolved actuators in the industry became more precise in the price of more complexity [7], [12]. To introduce new robotic platforms to address the demands of the modern era, proper actuators and materials should be used to achieve the required specifications of the robotic platforms [13]. Shape memory alloys (SMA)[14], piezoelectric[15], Electrostatic MEMS[16], and hydraulic/pneumatic actuators[17] are among the widely used actuators to design better robots.

Piezoelectric ceramics and thin films have been used extensively to develop various actuators that are proper to use in robots and end effectors [18]. The precise control on the movement of piezoelectric 
actuators, a high-power density, and a fast response time are the main benefits of piezoelectric actuators [19][20] [21].Piezoceramics can induce developing various types of actuators, still requires complicated micro-displacement transmission mechanism, and assembling of miniaturized actuators is challenging [22]. The high voltage required for piezoelectric material actuation is another drawback of this family of actuators that can delimit their applications in the fields of biostudy and surgery [23].

SMA actuators use SMA wires or thin films as the actuating element. This simple mechanism can produce large force and stroke [24]. While various actuators can be developed with SMA wires and thin films, assembling of them is more challenging when the size of the devices is smaller than millimeter scale [25]. Complexity of the required heating/cooling systems alongside slow response are delimiting additional drawbacks of the SMA actuators [26] [27]. The proper and expensive thermal control system is essential [28][29]. The heating requirement for SMA elements of actuators can limits the application of these actuators to manipulate sensitive biospecimen and live tissues due to lateral thermal damage [30][31].

Electrostatic MEMS actuators are mainly based on the silicon microfabrication process[32]. The wellestablished fabrication process made this group of actuators an ideal and cost effective actuator as 2D structures and a good candidate for miniaturization [32] [33]. The challenges in proper packaging and isolation of MEMS actuators are the main drawbacks of this family of actuators [34]. All aforementioned actuators are developed based on the hard silicon materials. This material feature is a drawback that make a proper soft material encapsulation essential for these actuators for fragile sample manipulation, which will increase their complexities[35][36]. Hydraulic and pneumatic actuators and end effectors are another family of widely developed actuators [37]. These actuators have the problem of miniaturization and assembling for the smaller size of actuator [38]. They use tubing to energize the actuator that remove the risk of wiring, high voltage, and high temperature damages to the live tissues [39]. One of the main benefits of this family of actuators is their compatibility with soft materials [40].

Choosing proper material, to fabricate a new robotic platform and their robotic end effectors, is an essential step to design new level of device [41]. Soft materials can be an alternative for hard polymers and metals, which using them allows robotic end effectors to mimic the properties of soft tissues and biological actuators[40][42]. This allows surgeons and researchers to delicately handle live biological material[43]. Biocompatible, elastomers are among good candidates for soft robotic applications.

After determining the required amount of force, working media, and the required method of transferring force to the specimens, the proper micromanipulator should be developed[44]. In this paper, we have used soft material molding method to fabricate monolithic PDMS based three finger-like columns on a deformable membrane to conform two different types of microhand-like manipulators. The bending characterization of the microhands was investigated successfully by using optical microscope imaging. The force production characterization was executed using AE-800 series piezoresistive microforce sensor. The finite-element method simulation was performed by using ABAQUS 6.12 software. The simulation results are used as a design tool for future optimization and designs. To approve the ability of the developed devices to manipulate fragile biospecimen, a live ant was grabbed, held, and released successfully without any damage.

\section{Results}

\section{Design and Mechanism:}


The idea to achieve a 3D microhand-like structure in this research is to form a micro scale gripper that can close and open by adjusting pressure in a cavity. The main part of the designed hydraulic/pneumatic 3D microhand is created from a combination of a $150 \mu \mathrm{m}$ PDMS based membrane placed on the top of a cylindrical void and three columns, which are placed on the top of the membrane as fingers. The void, membrane, and three fingers form the monolithic gripper. There have been two different designs as it is depicted in fig.1. The design pictured in fig.1.a is compatible with microfluidic systems, while the one in fig.1.b is designed to be used directly on the tip of a syringe.

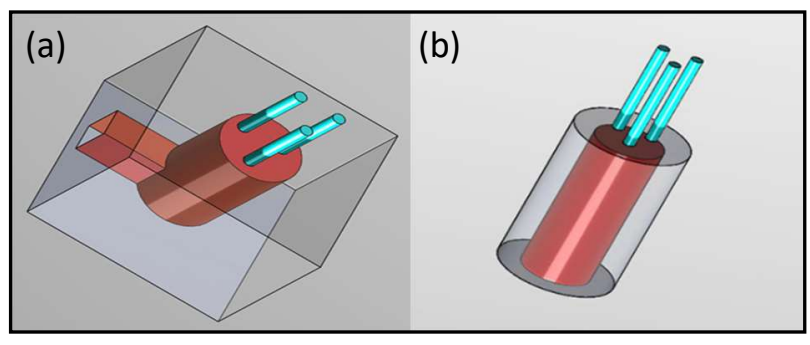

Figure 1. The designed microhand grippers. (a)Square shape device matched with microfluidics. The channel connected to the central void can be seen in this image. (b) The cylindrical device designed to be placed on syringe (requires an extra part and an assembling step).

As it is depicted in fig. 2 the fingers are placed on a deformable membrane. By deforming the membrane's shape into being concave or convex, the three fingertips on the top can cause opening or closing. Thus, the internal pressure of the cavity should be higher than the exterior's pressure to create convex membranes and lower for concave membranes. The deformation of the membrane can be calculated, either analytically or numerically, based on the amount of pressure. The deformation mechanism based on this pressure difference is independent of membrane geometries. An approximate for maximum displacement at the center of a circular membrane with the radius of $a=L x / 2$ is[45]:

$$
W_{0}=\left(p(a)^{4}\right) /\left(64 D_{\text {flex }}\right)
$$

This equation can give a very good insight about the membrane deflection and show the effects of static pressure ( $p$ ), dimensions and geometry (a) and material rigidity $\left(D_{\text {flex }}\right)$. By changing the membrane thickness, membrane diameter, or material stiffness, it is possible to have devices with different functional features. In equation 2, the deflection at ( $r$ ) can be calculated[45].

$W(r)=\left[\left(p(a)^{4}\right) /\left(64 D_{\text {flex }}\right)\right]\left(1-(r)^{2} /(a)^{2}\right)$

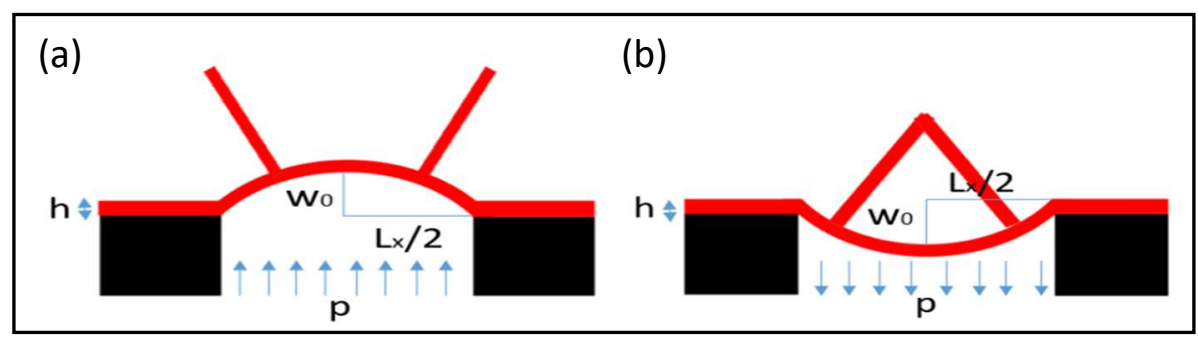

Figure 2. The fingers placed on the membrane and the opening/closing mechanism. (a) Opening the fingers. (b) Closing the fingers.

To choose the proper parameters for each types of these microhands, alongside with the application, the fabrication feasibility based on the fabrication method should be take in to account. To choose the 
diameter of each finger, which is $300 \mu \mathrm{m}$, the possibility to prepare the required molds in soft material molding method is the main parameter. Length of the fingers are $1.5 \mathrm{~mm}$ for microfluidic compatible device and are $2 \mathrm{~mm}$ for cylindrical microhands. These length was chosen based on the required size of the devices and the required produced force. The longer the finger, formed a bigger device and caused an smaller produced force. The diameter of the membrane affects the amount of the deformation. The diameter of the membrane is $1.8 \mathrm{~mm}$ for the cylindrical devices and $2 \mathrm{~mm}$ for the microfluidics compatible ones. The device with the smaller membrane bend more for the same amount of injected water.

\section{Fabrication:}

The micro-hand structure is fabricated from polydimethylsiloxane (PDMS) through soft material molding [46][47]. The required molds for this process were fabricated using a stereolithography 3D printer. To finalize the fabrication of 3D printed molds, they were exposed to light curing, thermal curing for 72 hours at $65^{\circ} \mathrm{C}$ in the oven, and the silane gas as the surface treatment, respectively. Siliconization can make the surface more hydrophobic through the inclusion of silane gas[48][49]. The two parts of the 3D printed molds are illustrated in the figure.3. In figure.3.(a,b) the molds for the cylindrical device are displayed, while in figure.3.(c,d) the molds for the square shape device are shown. For both types of devices, the first part of molds (shown in 3.a,c) were designed with three holes to form the three fingers, while the second part of molds (shown in 3.b,d) were responsible to form the central void as cavity and the membrane where the fingers are placed on. The two parts of molds for each device form an encapsulated closed volume. For PDMS the polymer and initiator were mixed with the (15:1) ratio to achieve a very soft and deformable material. The curing of PDMS was executed in one week at $25^{\circ} \mathrm{C}$. The combination of hydrophobic surface of the molds and a very flexible soft-material made it possible to remove the cured PDMS from the molds.

The fabrication steps for the Microhand are pictured in figure 4. To form the devices, the mixed liquid PDMS was poured into the first part of the molds, which contain three holes. The second part of the molds was covered by liquid PDMS(fig 4.a,b). Then they were placed in a vacuum to remove all the bubbles from the liquid. The two parts of the molds were closed (fig 4.c). Finally, the container of liquid PDMS that contains the molds with encapsulated liquid PDMS inside of them was placed in the vacuum to remove all the residual entrapped bubbles. After leaving the molds for one week at room temperature $\left(25^{\circ} \mathrm{C}\right)$, the two pieces opened, and the cured PDMS was removed slowly (fig 4.d). Because the cured PDMS was very soft with $1 \mathrm{MPa}$ young modulus, it was possible to remove it from the molds without damages. The SEM image of the formed three-fingered microhand is depicted in figure 5.

The final step of the fabrication was to assemble the microhand's parts and finalize the device. For the square shape device, the previously fabricated structure was bonded on a plate of cured PDMS by using oxygen plasma treatment and water as the bonding liquid[48][49]. When the water evaporates eventually, the strong hermetic bond formed between two pieces of cured PDMS, and the whole cavity and device formed successfully. For the cylindrical structure, a special connector designed, and 3D printed that was matched with the standard syringe tips, and the cured PDMS based microhand was attached to the $3 \mathrm{D}$ printed piece to finalize the device. The parts and finalized devices are shown in figure 6 . 


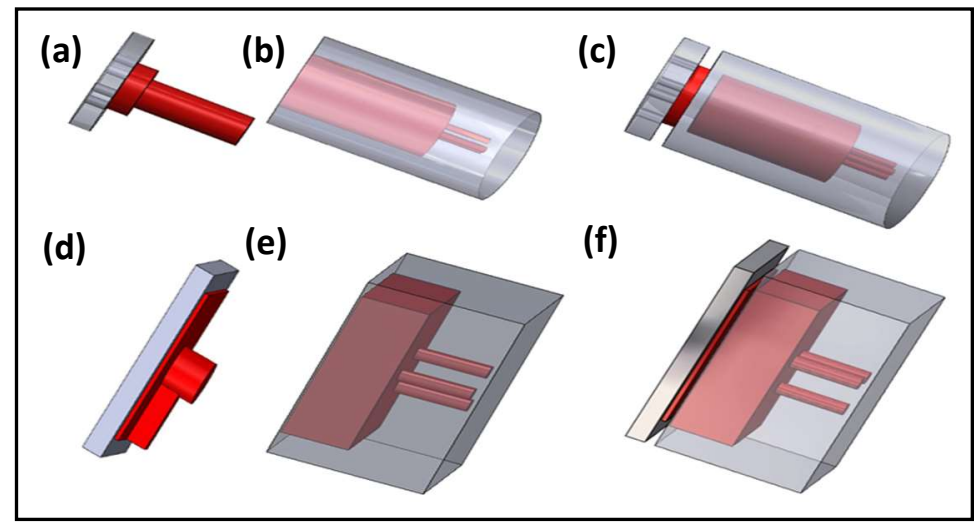

Figure. 3. The schematic model for 3D Printed Molds. a,b) The mold cap and mold for cylindrical microhand. c) The closing main part and cap of the mold to shape the whole mold for the cylindrical microhand. $d, e$,$) The mold cap and mold for square shape$ microhand. f) The closing main part and cap of the mold to shape the whole mold for the square shaped microhand. The red color shows the internal surfaces of the molds. These surfaces will be in touch with the liquid PDMS and will shape microhands.

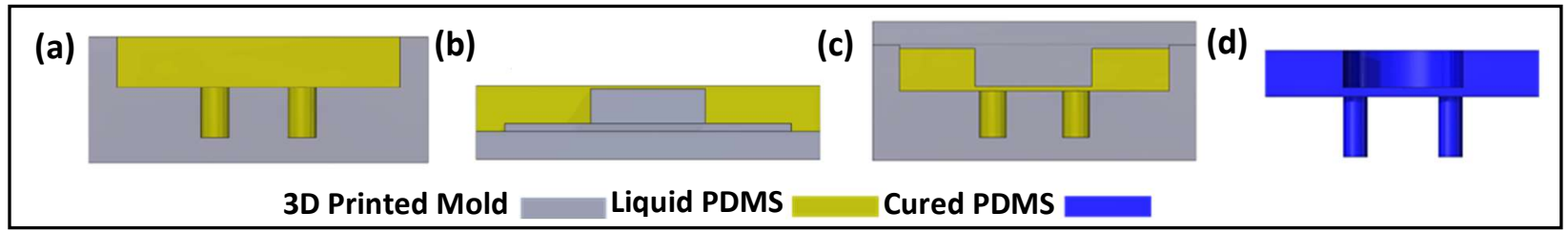

Figure.4. Microhand Fabrication Steps. a,b) The 3D printed mold was filled with and debubbled liquid PDMS, and the mold cap was covered with the same PDMS. c) Closing the mold and the mold cap. The liquid and debubbled PDMS was encapsulated in the 3D printed parts. As it can be seen, in this step the parts shown in (a) and (b) and filled and covered with PDMS are closed together to form the encapsulated area. d) PDMS was cured at $25^{\circ} \mathrm{C}$ in one week. The cured PDMS was removed from the $3 D$ printed molds and the functional part of the microhand was formed.

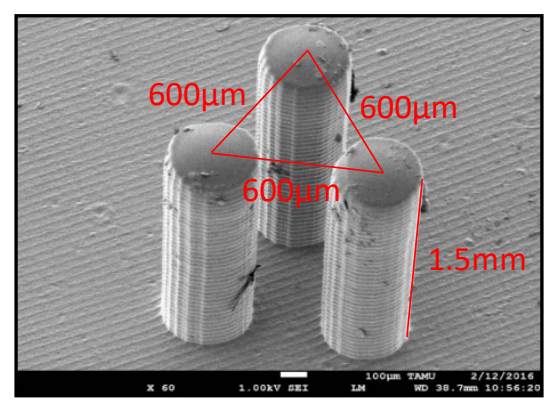

Figure.5.SEM image of three fingers. The diameter of each finger is $300 \mu \mathrm{m}$ and the center-to-center distance between each two fingers is $600 \mu \mathrm{m}$. The Triangle in the image shows the center-to-center distances between fingers. The length of each finger is $1.5 \mathrm{~mm}$ for the microfluidics compatible device. The length of fingers are $2 \mathrm{~mm}$ for cylindrical devices.

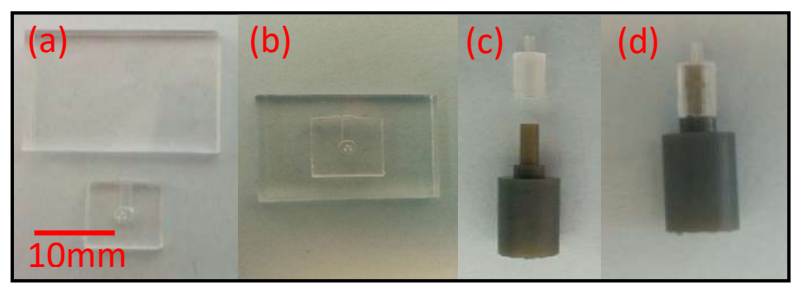

Figure.6. Assembling and finalizing the microhands. a) Square-shape microhand's elements. b) Finalized Square-shape microhand. c) Cylindrical microhand's elements. d) Finalized cylindrical microhand. 


\section{Characterization:}

\section{Deflection Characterization:}

The deflection characterization of microhands was perfromed using precise microliter syringes under an optical microscope. To characterize the mount of deflections, the micro-hand was placed under the microscope. The deflection-volume graphs were obtained by changing the injected water volume into the microhand's cavity. The deflection measurements were performed on optical images by using the software of Nikon Microscope. The side view of the bent fingers and the measured angle are depicted in figures $7(a, b)$.

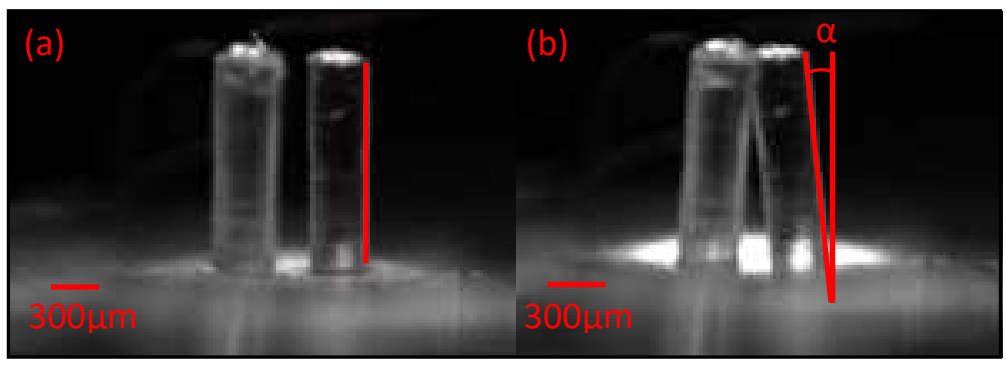

Figure.7. The sideview of fingers and the sideview of bending fingers and bending measurement. a) The side view of the microhand's fingers. $b$ ) The sideview of the bending finger and the measured angel of bending $(\alpha)$.

The bending-volume experimental measurements for the square-shaped devices are shown in figure 8 . The bending-volume experimental measurements for the cylindrical-shaped devices are depicted in figures $9(a, b)$. While figure 9 (a) contains the values for both opening and closing regimes, figure 9 (b) only contains the data of the opening regime on a broader range of injected volume changes.

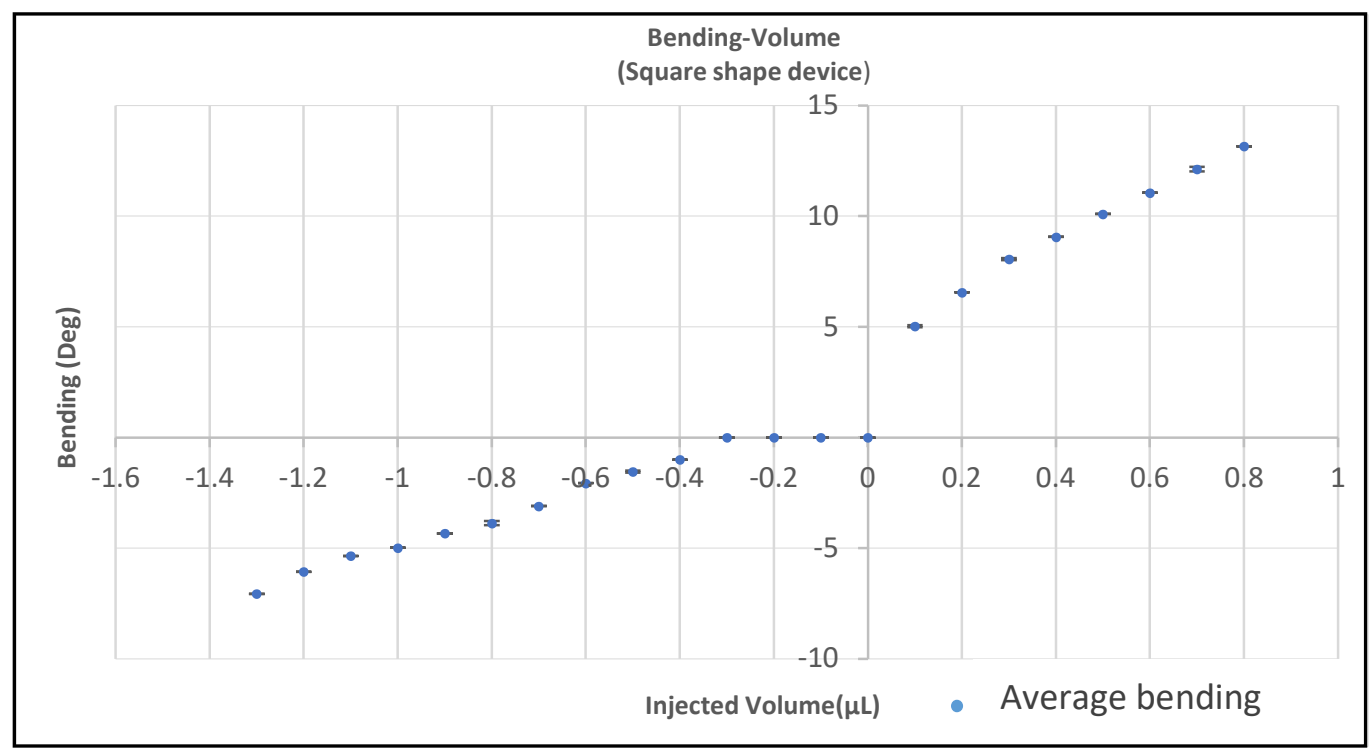

Figure.8. The square-shaped device bending characterization. The bending of the finger measured under an optical microscope. The angle $(\alpha)$ was measured as a function of the injected volume of water in the cavity. The negative angles are representing the angles during closing regime. 


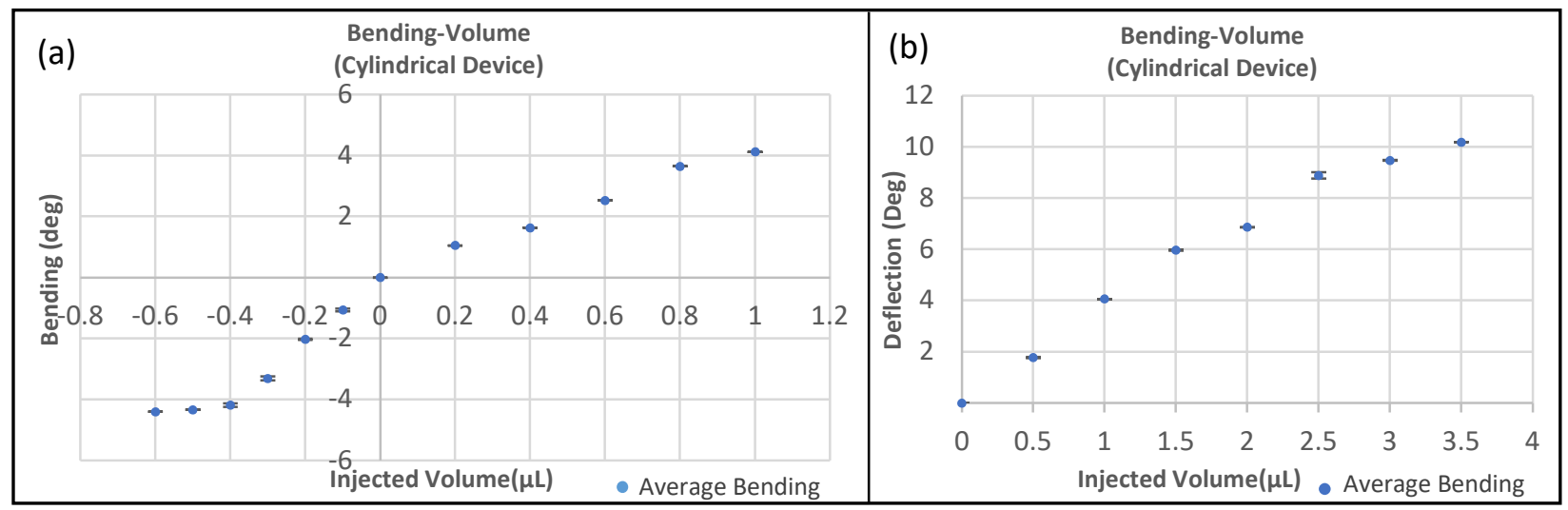

Figure.9. The cylindrical-shaped device bending characterization. (a)The bending of the finger was measured under an optical microscope. The angle $(\alpha)$ changings are shown versus the changes of injected volume of water in the cavity. The negative angles are representing angles during closing regime. (b)The bending of the finger was measured under an optical microscope. The angle $(\alpha)$ changes are shown versus the change of the injected volume of water in the cavity just for the opening regime.

\section{Force Characterization:}

To measure the produced forces of the two types of microhands, AE-800 microforce sensor was used. This cantilever-like microforce sensor works based on a piezoresistive mechanism. For effective measurement to avoid any unwanted misplacement during the force measurement, the sensor and the microhand were placed under a stereoscope. To ensure the proper measurement, the gap between the microhand's finger and the sensor's cantilever should be zero. Also, the overlap between the finger and the cantilever should be adjusted precisely to a specific $300 \mu \mathrm{m}$ amount and should be the same for all the experiments. In figure 10, the optical images of the microhand's finger and the sensor's cantilever can be seen.

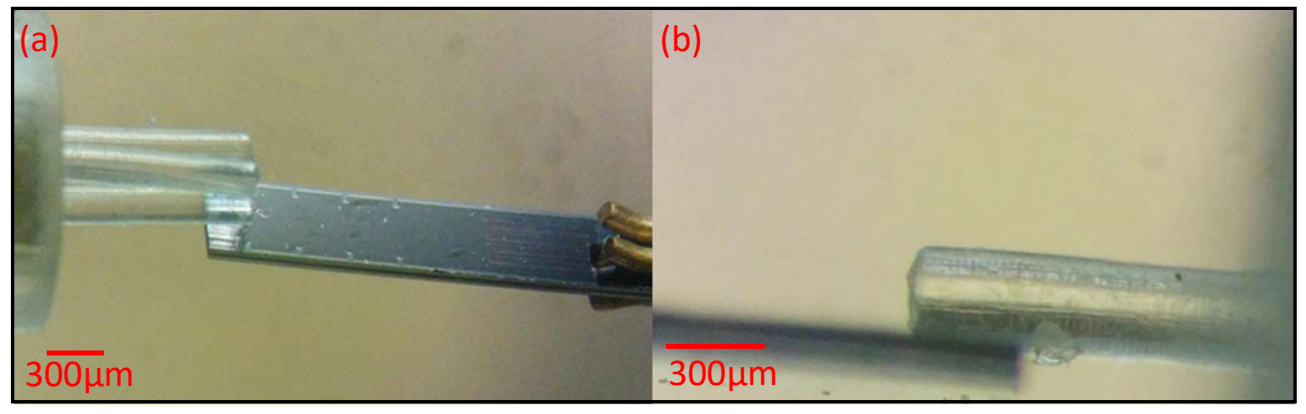

Figure.10. Force measurement by using AE-800 sensor. a) An optical image of the microhand and the force sensor. The suggested placement of the microhand and the sensor. b) The side view of a finger and the cantilever of the sensor.

The force-volume measurements for the square-shaped devices are shown in figure 11. 


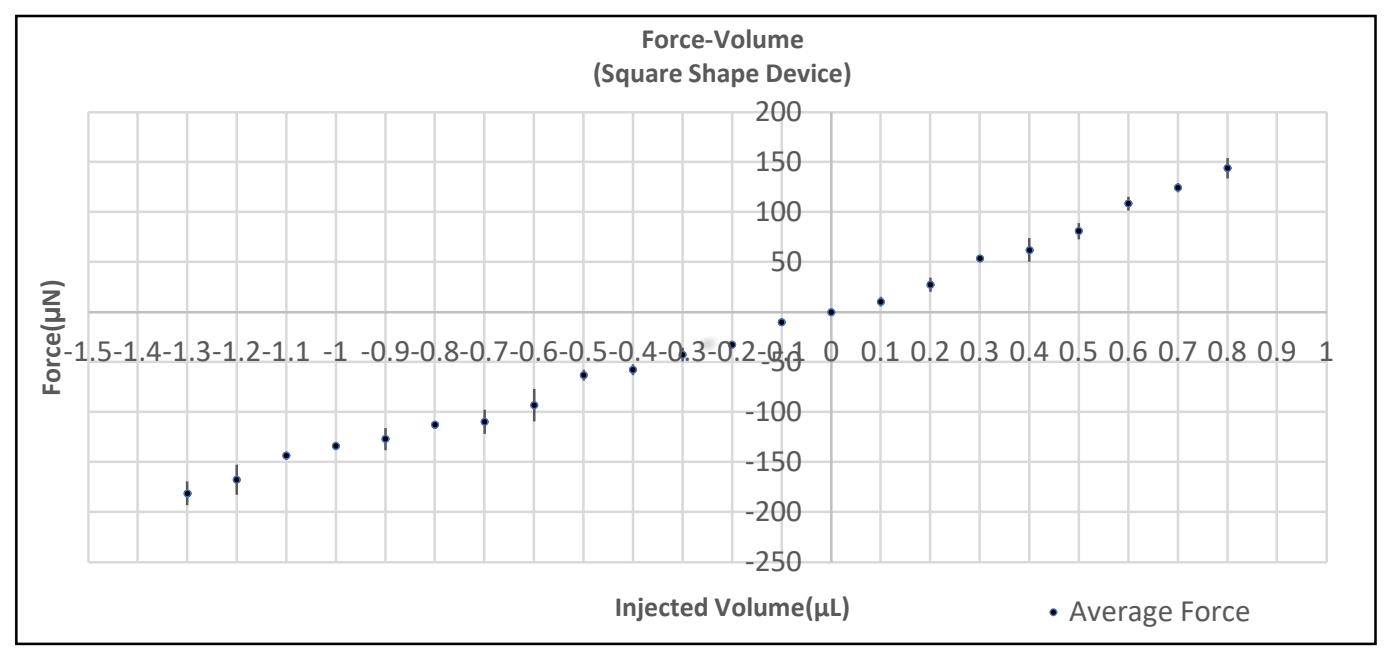

Figure.11. The square-shaped device force measurement. The force produced by one finger of the square-shaped microhand is measured under an optical microscope by using an AE-800 piezoresistive force sensor. The produced force changes are shown versus the changes of injected volume of water in the cavity. The negative forces are representing the produced forces during closing regime.

The force-volume measurements for the cylindrical-shaped devices are depicted in figures 12(a,b). While figure 12(a), contains the force values in both opening and closing regimes, figure 12(b) just contains the data for the opening regime on a broader range of volume changes.

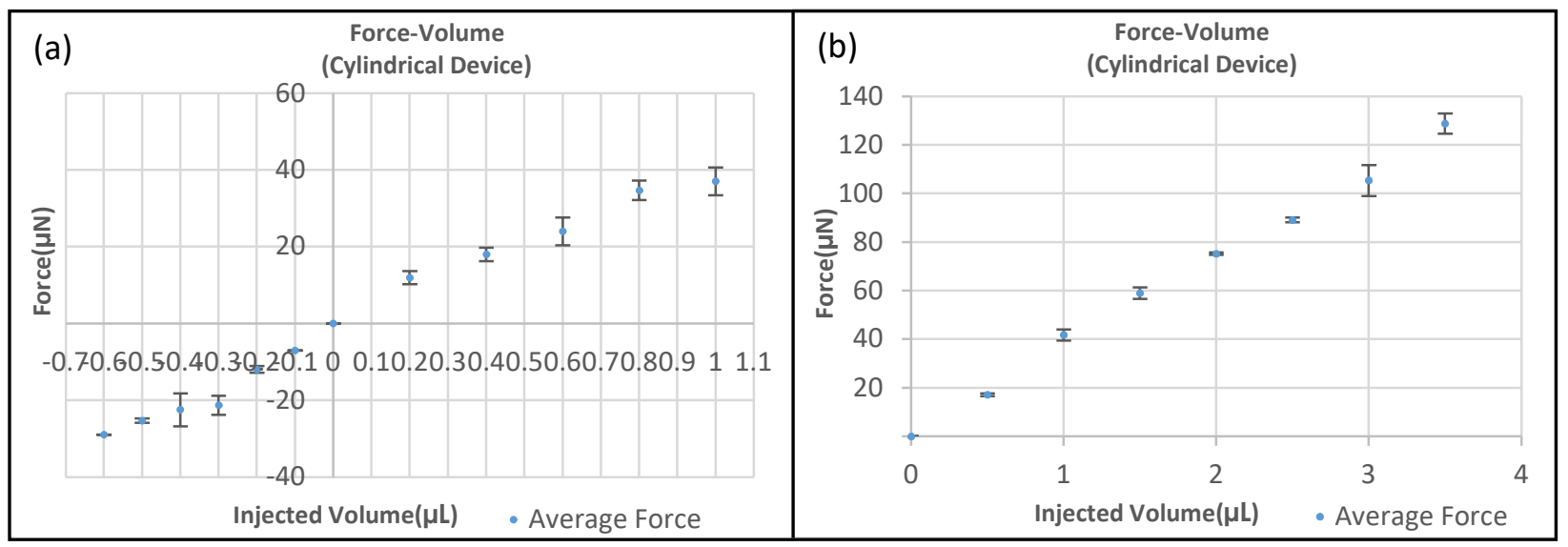

Figure.12. The cylindrical-shaped device force measurement. (a) The force produced by one finger of the cylindrical-shaped microhand is measured under an optical microscope by using an AE-800 piezoresistive sensor. The produced force changes are shown versus the changes of injected volume of water in the cavity. The negative forces are representing the produced force during closing regime.(b) The force produced by one finger of the cylindrical-shaped microhand is measured under an optical microscope by using an AE-800 piezoresistive sensor. The produced force changes are shown versus the changes of injected volume of water in the cavity.

\section{Simulation and Modelling:}

The finite-element software ABAQUS 6.12 was utilized to build a 3D model for simulating the hydraulic actuation of microhand devices. A slightly compressible Gent hyperelastic free energy, having two parameter empirical constitutive model, is used for the hyperelastic volumetric and isochoric isotropic terms of the isotropic part of constitutive model. The advantage of the model is to capture strain-stiffing at large strains, which are experimentally observed in soft materials. We separate the volumetric and deviatoric part of the constitutive equation for the finite element implementation to avoid numerical 
problems such as element locking. $W_{V}(J)$ denotes a purely volumetric and $W_{D}\left(\overline{I_{1}}\right)$ represents its deviatoric contribution represented by the slightly compressible Gent hyperelastic model given by,

$\mu$ is the shear modulus, $J_{m}$ is material constant, and $K$ is the bulk modulus. The uniaxial experimental data is fitted, and the material properties are $\mu=0.4 M P a, J_{m}=5.5$ and $\mathrm{K}=2000$.

The deformation results from simulations are shown in figure 13. The displacement of the bottom surface of the model was constrained since the model was bonded horizontally on a plate of PDMS. Applied

$$
\begin{aligned}
& W_{D}\left(\overline{I_{1}}\right)=-\frac{\mu J_{m}}{2} \ln \left(\frac{J_{m}-\overline{I_{1}}+3}{J_{m}}\right), \\
& W_{V}(J)=-\frac{K}{2} \ln (\mathrm{J})+\frac{K}{2} \frac{J^{2}-1}{2}
\end{aligned}
$$

hydraulic pressures were set as pressure load which acts in the normal direction on the hydraulic channel. General surface-to-surface contacts were set up for the three fingers of the models in figure 9.

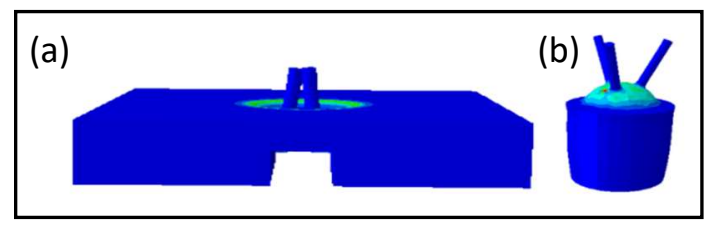

Figure 13. 3D model and simulation results. a) Simulation of deformation of the fingers in closing regime, in a square-shaped device b) Simulation of deformation of the fingers in opening regime, in a cylindrical-shaped device.

While the material parameters obtained from uniaxial experiment, other parameters of the microhand like dimensions of the fingers and membrane were determined directly from the devices. The comparisons of the simulation and experimental measurements are depicted in figures $14(a, b)$. These graphs show that the simulation and experimental results are in a very good agreement, which means the 3D model is appropriately developed and can later be used in the design process of the devices.

Further development of this simulation can provide a powerful tool for the design and optimization of the parameters of required microhands in the future.

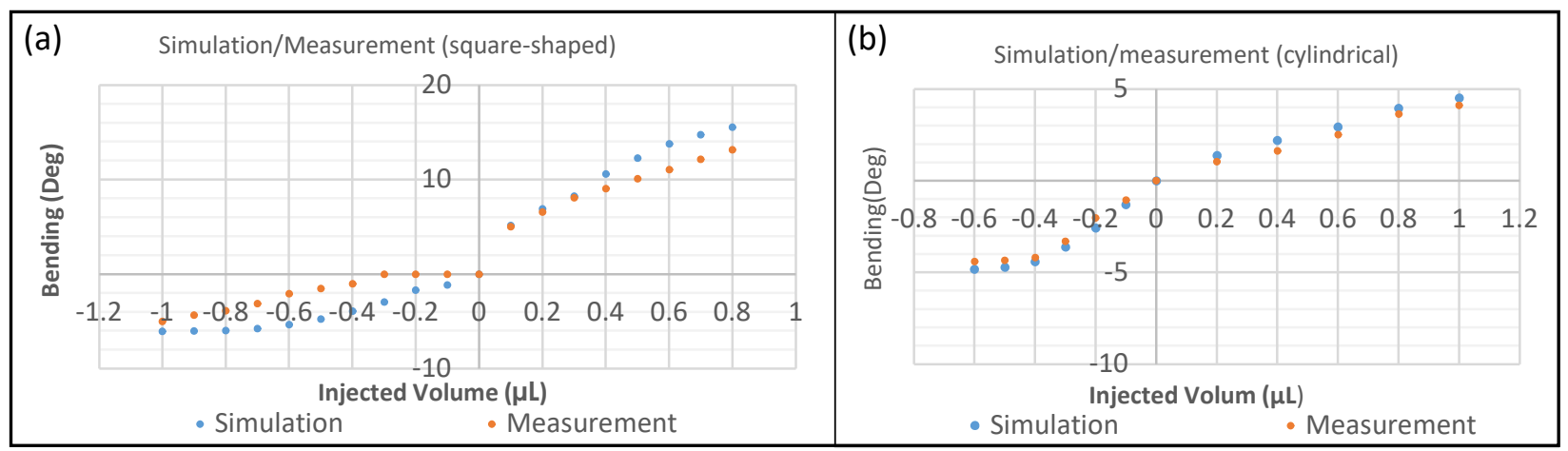

Figure.14. Comparison of the simulation and experimental measurement. (a) The comparison between simulation and measurement for the square-shaped device in the closing regime. The simulation and experimental measurement show almost the same slope as depicted in the figure. (b) The comparison between simulation and measurement for the cylindrical-shaped device in opening and closing regimes. The simulation and measurement show almost a same slope as depicted in the figure. 


\section{Biospecimen manipulation:}

To prove the hypothesis that these microhands can manipulate a biological fragile sample precisely, the manipulation of an ant was performed under a microscope. The length of the ant was almost $3 \mathrm{~mm}$ and the average thicknes if its body is around $400 \mu \mathrm{m}$. The microhand was able to grab, hold, and release a live ant without physically damaging it. So, by adjusting the pressure and the finger location, the manipulating of the fragile specimen was performed successfully. The manipulation of an ant is shown in figure 15. For this purpose, $543 \mu \mathrm{N}$ of force was induced to the ant by using square-shaped device and $87 \mu \mathrm{N}$ was induced to the same insect by using cylindrical device. Both numbers were the maximum force the devices could produce.

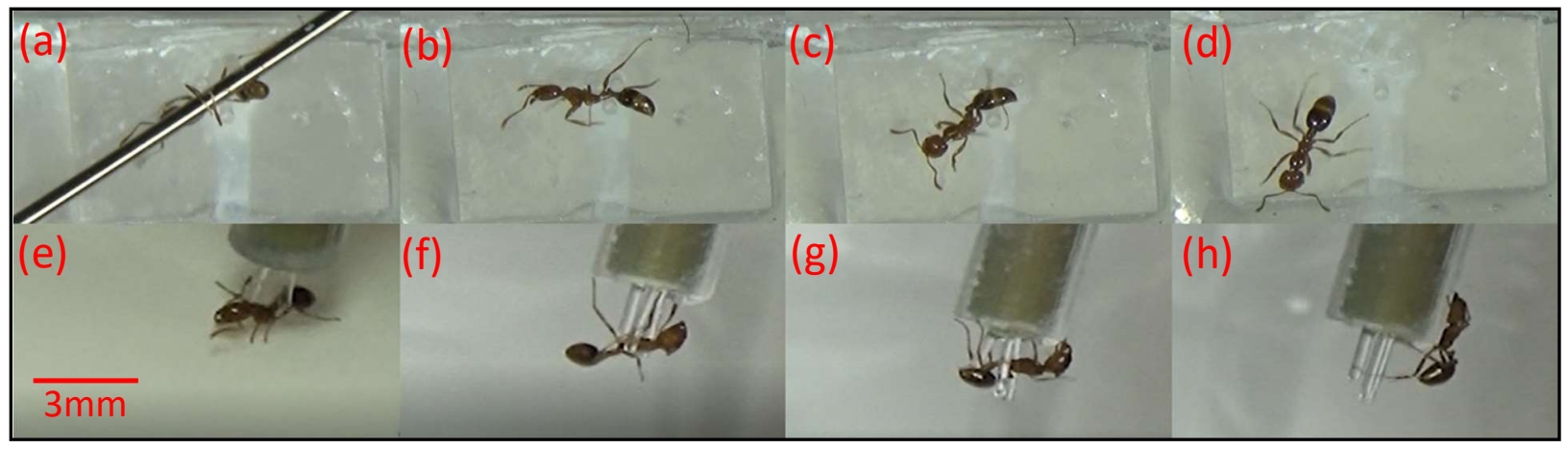

Figure.15. Manipulation of a live insect. a) Start of the grabbing of the ant. A needle is used to hold the insect on the top of gripper. b) Successful handling of a live ant by using a square-shaped device. c) Opening of the gripper to release the ant. d) The ant released and walked away. e) Start of handling of the grabbing of the ant. f) Successful handling of the live ant with a cylindrical-shaped device. g) Opening of the gripper to release the ant. h) The ant released and walked away. (A same ant was used during the experiments).

\section{Discussion:}

The innovative microhand-like devices were developed by using soft material molding technique with 3D

printed molds. The microhands made from PDMS, with their produced force and motion precisely controllable, made this innovative manipulator a practical option for biospecimen handling.

As mentioned in the fabrication part, the proposed microhand can be fabricated in a single step molding of PDMS. The innovative, encapsulated, molding technique was used to fabricate two different types of microhands introduced in this paper. The actuation mechanism is the bending of a flexible membrane due to pressure difference on two surfaces of the membrane. As it can be concluded from equation (1) the higher-pressure difference or a wider membrane will cause more deformation. At the same time, a thicker membrane or a stiffer material can limit the deformation of the membrane. The fingers of the microhand were placed on this flexible membrane.

The precision in the bending of the fingers is revealed from characterization. The wide range of the bending of the fingers, alongside the precise controllability of this bending, confirms the ability of the microhand to manipulate a wide range of fragile objects. The bending angle for each finger was ranged from 8 degrees of closing to 13 degrees of opening for square-shaped devices, and from 4.4 degrees of closing to 10.2 degrees of opening for cylindrical-shaped devices. The precision of the fingers' deflection highly depends on the microliter syringes' precision and the precision in controlling the syringes. Each step of the injected volume into the cavity of a microhand was $0.1 \mu \mathrm{L}$ in this study. And the minimum step 
of the measured bending angle was 0.4 of a degree for the square-shaped devices and 0.1 of a degree for the cylindrical-shaped devices.

From the force measurement, it revealed the maximum produced force of the microhands is smaller than $1 \mathrm{mN}$, which means they are suitable for biospecimen handling [50]. The maximum absolute produced force of a single finger in a square-shaped device is around $181 \mu \mathrm{N}$, which means three fingers can impose around $543 \mu \mathrm{N}$ on a specimen. Similarly, the experiments revealed the absolute maximum force a finger can produce in a cylindrical-shaped device is $30 \mu \mathrm{N}$, which is equal to $90 \mu \mathrm{N}$ for three fingers. The forcevolume ratio, which can be described as the absolute force divided to the volume of the gripper and actuator, for the square-shaped microhand is $1.13 \mathrm{mN} / \mathrm{mm}^{3}$ and for the cylindrical-shaped device is 0.141 $\mathrm{mN} / \mathrm{mm}^{3}$. As references, other soft grippers have maximum absolute produced force of $50 \mathrm{mN}, 3 \mathrm{mN}$, and $2.2 \mathrm{mN}$, and when the size of the grippers take into account the force-volume ratio is $0.78,3.3$, and 0.047 $\mathrm{mN} / \mathrm{mm}^{3}$ respectively[51][52][53]. Both the produced force and force-volume ratio are higher or comparable with other soft manipulators in literature [54].

The simulation of the microhand revealed that the 3D model in the finite-element simulation can be used successfully to predict the actuation of the fingers. The results of this simulation can be used as a design tool to optimize the required parameters of the device. Disagreements between the simulation and experimental measurements can be mainly because of microscale variation of used PDMS in the membrane, soft material variations during the fabrication, or fabrication method resolutions. Absorption of the hydraulic pressure by different parts like soft tubes that were used for transfer pressure from the syringes to the device or soft surfaces except the membrane can be other issues that cause differences between simulations and experimental measurements. While more refined models can cause better prediction of microhand moving manner, the current simulation shows proper prediction of the produced force of the fingers.

\section{-Methods}

Microhand Fabrication. As depicted in figure 1, the fabrication process was started by 3D printing of the proper molds by using Perfactory Microprinter made by Envisiontech. After Printing the molds, the lightcuring of the molds was done. And they be left in the oven at $65^{\circ} \mathrm{C}$ for 72 hours. After that, the molds were covered by silane gas, because the untreated molds are stickier for PDMS. Because the molds are combined from two different pieces, they will make an enclosed space after assembling. So, for fabricating PDMS based structure, the molds at first should be filled by PDMS and remove the bobbles in a vacuum, then the molds will be closed, and finally, they will be sunk in PDMS and again locate in the vacuum to remove bobbles for 30 minutes. The molds and PDMS were left at room temperature for one week and then the slowly cured PDMS based structures removed carefully from the molds. To shape up the final structure, the PDMS-based structure had been bonded to the surface of a plate from the same material and finally a piece of a tube connected to the channel to form the whole structure.

Opening and closing Characterization. For characterization, the opening and closing of the Micro-Hands and achieving "Deflection-Injected Volume" curve, the micro-hand was placed under an optical microscope and was connected to a microliter syringe. By changing the injected volume in the cavity and measuring the finger's deflection under the microscope the characterization performed successfully and deflection- injected volume curve was achieved.

Force Measurement. Ae-800 microforce sensor is a cantilever-like piezoresistive one used to measure the force produced by the fingers of microhand. For proper placing of the finger over the sensor, the whole process was done under an optical stereoscope. 
Simulation. As mentioned, ABAQUS 6.12 was used to build up a 3D model for simulating the hydraulic actuation of the micro hand. The model used 376,938 tetrahedral elements and was consisted of 10-node modified hybrid tetrahedral elements (ABAQUS C3D10MH). Modified tetrahedral elements provided a good convergence rate and prevented volumetric locking. Hyperelastic material models were highly capable of describing the nonlinear response of complex materials. With the experiment's data fitted and analyzed, the PDMS was modeled as an incompressible and isotropic Gent model, which was satisfied with convergence and solution stability. The Gent model with the isotropic condition was applied and the material properties were $\mu=0.4 M P a$ and $J_{m}=5.5$ for the studied PDMS material.

Grabbing bio-samples. An ant was chosen as a biospecimen in this project, grabbed, and manipulated successfully as is shown in figure 10. For this purpose, the ant was placed on an ice pack and halted due to low temperature. This made it easier to grab the insect. The grabbing of the biospecimen was performed under a Nikon Stereoscope. After grabbing the ant, it was separated from the ice pack in the way it warmed up and started to move again. After 1 minute of holding the moving insect, it was released, and no injuries were observed. The grabbing and manipulation of the ant was performed with both kinds of devices that were developed and reported in this article.

\section{References}

[1] A. M. Okamura, “NIH Public Access,” vol. 19, no. 1, pp. 102-107, 2009, doi: 10.1097/MOU.0b013e32831a478c.Haptic.

[2] M. J. Mack, “Minimally Invasive and Robotic Surgery,” Oppor. Med. Res., vol. 285, no. 5, 2001.

[3] N. R. Sinatra, C. B. Teeple, D. M. Vogt, K. K. Parker, D. F. Gruber, and R. J. Wood, "Ultragenttle Manipulation of delicate structure using a soft robotic gripper." Science Robotic, pp. 1-11, 2019.

[4] D. M. Vogt et al., "Shipboard design and fabrication of custom 3D-printed soft robotic manipulators for the investigation of delicate deep-sea organisms," PLoS One, pp. 1-16, 2018.

[5] K. T. Andrzejewski, M. P. Cooper, C. A. Griffiths, and C. Giannetti, "Optimisation process for robotic assembly of electronic components," Int. J. Adv. Manuf. Technol., pp. 2523-2535, 2018.

[6] B. J. Schroer and E. F. Stafford, "Issues in Using Robots for Electronics Assembly," Robotics, vol. 2, no. 3, pp. 225-235, 1986, doi: doi.org/10.1016/0167-8493(86)90031-8.

[7] V. Vitiello, S. Lee, T. P. Cundy, and G. Yang, "Emerging Robotic Platforms for Minimally Invasive Surgery," IEEE Rev. Biomed. Eng., vol. 6, pp. 111-126, 2013, doi: 10.1109/RBME.2012.2236311.

[8] C. Bergeles and G. Z. Yang, "From passive tool holders to microsurgeons: Safer, smaller, smarter surgical robots," IEEE Trans. Biomed. Eng., vol. 61, no. 5, pp. 1565-1576, 2014, doi: 10.1109/TBME.2013.2293815.

[9] K. C. Galloway et al., "Soft Robotic Grippers for Biological Sampling on Deep Reefs," Soft Robot., vol. 3, no. 1, pp. 23-46, 2016, doi: 10.1089/soro.2015.0019.

[10] J. Zhou, S. Member, S. Chen, Z. Wang, and S. Member, "A Soft-Robotic Gripper With Enhanced Object Adaptation and Grasping Reliability," IEEE Robot. Autom. Lett., vol. 2, no. 4, pp. 2287-2293, 2017, doi: 10.1109/LRA.2017.2716445.

[11] D. T. Pham and S. H. Yeo, "Strategies for gripper design and selection in robotic assembly.pdf," Int. J. Prod. Res., vol. 29, no. 2, pp. 303316, 1991, doi: 10.1080/00207549108930072.

[12] M. Verotti, A. Dochshanov, and N. P. Belfiore, "Compliance Synthesis of CSFH MEMS-Based Microgrippers," J. Mech. Des. Trans. ASME, vol. 139, no. 2, pp. 1-10, 2017, doi: 10.1115/1.4035053.

[13] M. Honarpardaz, M. Tarkian, J. Ölvander, and X. Feng, “Finger design automation for industrial robot grippers : A review," Rob. Auton. Syst., vol. 87, pp. 104-119, 2017, doi: 10.1016/j.robot.2016.10.003.

[14] M. Garcés-schröder, L. Hecht, A. Vierheller, M. Leester-schädel, M. Böl, and A. Dietzel, "Micro-Grippers with Femtosecond-Laser Machined In-Plane Agonist-Antagonist SMA Actuators Integrated on Wafer-Level by Galvanic Riveting," in Eurosensors, 2017, pp. 1-5, doi: 10.3390/proceedings1040385.

[15] Y. Yang, J. Lou, G. Wu, Y. Wei, and L. Fu, "Design and position / force control of an S-shaped MFC microgripper," Sensors Actuators A Phys., vol. 282, pp. 63-78, 2018, doi: 10.1016/j.sna.2018.09.021. 
[16] S. Bütefisch, V. Seidemann, and S. Büttgenbach, "Novel Micro-pneumatic actuator for MEMS.pdf," Sensors Actuators A Phys., vol. 9798, pp. 638-645, 2002.

[17] P. Moseley, J. M. Florez, H. A. Sonar, G. Agarwal, W. Curtin, and J. Paik, "Modeling, Design, and Development of Soft Pneumatic Actuators with Finite Element Method," Adv. Eng. Mater., vol. 18, no. 6, pp. 978-988, 2016, doi: 10.1002/adem.201500503.

[18] S. Chopra and N. Gravish, "Piezoelectric actuators with on-board sensing for micro-robotic applications," Smart Mater. Struct., vol. 28, no. 11, p. 10pp, 2019, doi: 10.1088/1361-665X/ab43fe.

[19] F. Filhol, E. Defaÿ, C. Divoux, C. Zinck, and M.-T. Delaye, "Resonance Micro-mirror excited by a thin-film piezoelectric actuator for fast optical beam scanning," Sensors Actuators A Phys., vol. 123-124, pp. 483-489, 2005.

[20] Q. Xu, "Precision position/force interaction control of a piezoelectric multimorph microgripper for microassembly," IEEE Trans. Autom. Sci. Eng., vol. 10, no. 3, pp. 503-514, 2013, doi: 10.1109/TASE.2013.2239288.

[21] N. T. Jafferis, M. Lok, N. Winey, G. Y. Wei, and R. J. Wood, "Multilayer laminated piezoelectric bending actuators: Design and manufacturing for optimum power density and efficiency," Smart Mater. Struct., vol. 25, no. 5, 2016, doi: 10.1088/0964$1726 / 25 / 5 / 055033$.

[22] D. H. Wang, Q. Yang, and H. M. Dong, "A monolithic compliant piezoelectric-driven microgripper: Design, modeling, and testing," IEEE/ASME Trans. Mechatronics, vol. 18, no. 1, pp. 138-147, 2013, doi: 10.1109/TMECH.2011.2163200.

[23] M. Karpelson, G.-Y. Wei, and R. J. Wood, "Driving high voltage piezoelectric actuators in microrobotic applications," Sensors Actuators A Phys., vol. 176, pp. 78-89, 2012, doi: 10.1016/j.sna.2011.11.035.

[24] C. Calhoun, R. Wheeler, T. Baxevanis, and D. . Lagoudas, "Actuation fatigue life prediction of shape memory alloys under the constantstress loading condition," Scr. Mater., vol. 95, pp. 58-61, 2015, doi: 10.1016/j.scriptamat.2014.10.005.

[25] A. Nespoli, S. Besseghini, S. Pittaccio, E. Villa, and S. Viscuso, "The high potential of shape memory alloys in developing miniature mechanical devices : A review on shape memory alloy mini-actuators," Sensors Actuators A Phys., vol. 158, pp. 149-160, 2010, doi: 10.1016/j.sna.2009.12.020.

[26] J. W. Sohn, G.-W. Kim, and S.-B. Choi, "A State-of-the-Art Review on Robots and Medical Devices Using Smart Fluids and Shape Memory Alloys," Appl. Sci., vol. 8, no. 1928, pp. 1-21, 2018, doi: 10.3390/app8101928.

[27] C. R. Knick, D. J. Sharar, A. A. Wilson, G. L. Smith, C. J. Morris, and H. A. Bruck, "High frequency, low power, electrically actuated shape memory alloy MEMS bimorph thermal actuators," J. Micromechanics Microengineering, vol. 29, no. 7, pp. 1-8, 2019, doi: 10.1088/13616439/ab1633.

[28] Z. Wang, G. Hang, J. Li, Y. Wang, and K. Xiao, "A micro-robot fish with embeded SMA wire actuaed flexible biomimetic fin," Sensors Actuators A Phys., vol. 144, no. 2, pp. 354-360, 2008.

[29] H. Asadi, M. Eynbeygi, and Q. Wang, "Nonlinear thermal stability of geometrically imperfect shape memory alloy hybrid laminated composite plates," Smart Mater. Struct., vol. 23, no. 7, pp. 1-13, 2014, doi: 10.1088/0964-1726/23/7/075012.

[30] S. Szykowny and M. H. Elahinia, "Heat Transfer Analysis of Shape Memory Alloy Actuators," in IMECE, 2006, pp. 1-8.

[31] Z. Liu, K. Xiao, Z. Hou, F. Yan, Y. Chen, and L. Cai, "Multifunctional Coating with Both Thermal Insulation and Antibacterial Properties Applied to Nickel-Titanium Alloy," Int. J. Nanomedicine, vol. 15, pp. 7215-7234, 2020, doi: 10.2147/IJN.S266247.

[32] S. Yang and Q. Xu, "A review on actuation and sensing techniques for MEMS-based microgrippers," J. Micro-Bio Robot, vol. 13, pp. 114, 2017, doi: 10.1007/s12213-017-0098-2.

[33] Z. Qiu and W. Piyawattanamatha, "New Endoscopic Imaging Technology Based on MEMS Sensors and Actuators," Micromachines, vol. 8, no. 210, pp. 1-27, 2017, doi: 10.3390/mi8070210.

[34] R. Ramesham and R. Ghafkian, "Challenges in Interconnection and Packaging of Microelectromechanical Systems ( MEMS )," in 2000 Proceedings. 50th Electronic Components and Technology Conference, 2000, no. Ic, pp. 666-675, doi: 10.1109/ECTC.2000.853230.

[35] H. Yang, M. Xu, W. Li, and S. Zhang, "Design and Implementation of a Soft Robotic Arm Driven by SMA Coils," IEEE Trans. Ind. Electron., vol. 66, no. 8, pp. 6108-6116, 2019, doi: 10.1109/TIE.2018.2872005.

[36] S. B. Kordmahale and J. Kameoka, "Smart Soft Actuation System," Ann. Mater. Sci. Eng., vol. 2, no. c, pp. 2-3, 2015.

[37] B. Gorissen, D. Reynaerts, S. Konishi, K. Yoshida, J. W. Kim, and M. De Volder, "Elastic Inflatable Actuators for Soft Robotic Applications," Adv. Mater., vol. 29, no. 43, pp. 1-14, 2017, doi: 10.1002/adma.201604977.

[38] M. De Volder and D. Reynaerts, "Pneumatic and hydraulic microactuators: A review," J. Micromechanics Microengineering, vol. 20, no. 4, pp. 1-18, 2010, doi: 10.1088/0960-1317/20/4/043001. 
[39] S. Ozbek, E. Foo, J. W. Lee, N. Schleif, and B. Holschuh, "Low-power, minimal-heat exposure shape memory alloy (SMA) actuators for on-body soft robotics," in Proceedings of the 2019 Design of Medical Devices Conference, 2019, pp. 1-5, doi: 10.1115/DMD2019-3287.

[40] A. Miriyev, K. Stack, and H. Lipson, "Soft material for soft actuators," Nat. Commun., vol. 8, no. 1, pp. 1-8, 2017, doi: 10.1038/s41467017-00685-3.

[41] D. Rus and M. T. Tolley, “Design, Fabrication and control of soft robots.pdf,” Nature, vol. 521, pp. 467-475, 2015, doi: $10.1038 /$ nature14543.

[42] D. Steck, J. Qu, S. B.Kordmahale, D. Tscharnuter, A. Muliana, and J. Kameoka, "Mechanical responses of Ecoflex silicone rubber: Compressible and incompressible behaviors," J. Appl. Polym. Sci., vol. 136, no. 5, pp. 1-11, 2019, doi: 10.1002/app.47025.

[43] F. Hartmann, M. Baumgartner, and M. Kaltenbrunner, "Becoming Sustainable, The New Frontier in Soft Robotics," Adv. Mater., vol. 2004413, pp. 1-32, 2020, doi: 10.1002/adma.202004413.

[44] Z. Samadikhoshkho, K. Zareinia, and F. Janabi-Sharifi, "A Brief Review on Robotic Grippers Classifications," in 2019 IEEE Canadian Conference of Electrical and Computer Engineering, 2019, pp. 10-13, doi: 10.1109/CCECE.2019.8861780.

[45] M. Bao, "Chapter 2-Mechanics of Beam and Diaphragm Structures," in Analysis and Design Principles of MEMS Devices, 2005, pp. 33114, doi: 10.1016/B978-044451616-9/50003-5.

[46] S. B. Kordmahale, J. Do, K. A. Chang, and J. Kameoka, "Low Cost and Piezoelectric based Soft Wave Energy Harvester," in MRS Advances, 2019, vol. 4, no. 15, pp. 889-895, doi: 10.1557/adv.2018.675.

[47] P.-J. Huang, S. Baghbani Kordmahale, C.-K. Chou, H. Yamaguchi, M.-C. Hung, and J. Kameoka, "Development of automated high throughput single molecular microfluidic detection platform for signal transduction analysis," in SPIE 9705, Microfluidics, BioMEMS, and Medical Microsystems XIV, vol. 9705, pp. 1-5, doi: 10.1117/12.2213259.

[48] A. P. Saghati, S. B. Kordmahale, A. P. Saghati, J. Kameoka, and K. Entesari, "Reconfigurable quarter-mode SIW antenna employing a fluidically switchable via," in 2016 IEEE Antennas and Propagation Society International Symposium, APSURSI 2016 - Proceedings, 2016 , pp. 845-846, doi: 10.1109/APS.2016.7696131.

[49] A. P. Saghati, S. B. Kordmahale, J. Kameoka, and K. Entesari, "Reconfigurable Quarter-Mode Substrate Integrated Waveguide Cavity Filter Employing Liquid-Metal Capacitive Loading," in 2015 IEEE MTT-S International Microwave Symposium, 2015, pp. 20-22, doi: 10.1109/MWSYM.2015.7166885.

[50] S. Shimomura, Y. Teramachi, Y. Muramatsu, S. Tajima, Y. Tabata, and S. Konishi, "Pinching and releasing of cellular aggregate by microfingers using PDMS pneumatic balloon actuators," Proc. IEEE Int. Conf. Micro Electro Mech. Syst., no. 3, pp. 925-926, 2014, doi: 10.1109/MEMSYS.2014.6765793.

[51] S. Konishi, F. Kawai, and P. Cusin, "Thin flexible end-effector using pneumatic balloon actuator," Sensors Actuators, A Phys., vol. 89, no. 1-2, pp. 28-35, 2001, doi: 10.1016/S0924-4247(00)00533-1.

[52] Y. Watanabe, M. Maeda, N. Yaji, R. Nakamura, and H. Iseki, “Tokyo Women 's Medical University," no. January, pp. 659-662, 2007.

[53] S. Wakimoto, K. Suzumori, and K. Ogura, "Miniature pneumatic curling rubber actuator generating bidirectional motion with one airsupply tube," Adv. Robot., vol. 25, no. 9-10, pp. 1311-1330, 2011, doi: 10.1163/016918611X574731.

[54] J. Paek, I. Cho, and J. Kim, "Microrobotic tentacles with spiral bending capability based on shape-engineered elastomeric microtubes," Scientific Reports, vol. 5. 2015, doi: 10.1038/srep10768.

\section{Author Contributions}

J.K conceived the idea of having a microgripper. S.BK designed and developed microhands, established the fabrication protocols, and performed characterizations. J.Q developed the finite element model for the simulation and future shape engineering, and performed the simulations. J.K and AH.M supervised the research, validated all the information, and revised the manuscript. All Authors co-wrote the manuscript. 